Hadron Structure '15

International Journal of Modern Physics: Conference Series

Vol. 39 (2015) 1560083 (8 pages)

(C) The Author(s)

DOI: $10.1142 /$ S2010194515600836

\title{
Hadron spin and external fields
}

\author{
O. V. Teryaev \\ Bogoliubov Laboratory for Theoretical Physics \\ Joint Institute for Nuclear Research \\ Dubna 141980, Russia \\ teryaev@theor.jinr.ru
}

Published 26 November 2015

\begin{abstract}
The interactions of hadron spin with gravity and electromagnetic field and their implication for hadron spin structure are considered. The extension of equivalence principle naturally leads to the closeness of vector meson magnetic moment to that of electroweak boson. This similarity is confirmed in the lattice QCD. The effective gravity in AdS/QCD allows to connect the $t$-dependence of GPDs (and therefore electromagnetic and gravitational form factors) with $k_{T}$-dependemce of TMDs. The possible cosmological implications of equivalence principle extension are discussed.
\end{abstract}

Keywords: Gravity; magnetic moment.

PACS Numbers: 04.25.Nx, 12.38.Aw

\section{Introduction}

The spin structure of hadrons is one of the fundamental properties of nonperturbative QCD. I consider here one particular aspect of this problem, corresponding to the spin interaction with external electromagnetic (EM) and gravitational fields. I will concentrate on the issues related to Equivalence Principle and its extension, mentioning only those selected problems of the vast field of interaction with EM fields which has some connection to that.

\section{Nucleon in the External Gravitational Field}

Here we follow ${ }^{1}$ in considering the action of electromagnetic and gravity fields in uniform manner. Let us start with the more common case of the interaction with electromagnetic field, described by the matrix element of electromagnetic current,

$$
M=\left\langle P^{\prime}\left|J_{q}^{\mu}\right| P\right\rangle A_{\mu} .
$$

This is an Open Access article published by World Scientific Publishing Company. It is distributed under the terms of the Creative Commons Attribution 3.0 (CC-BY) License. Further distribution of this work is permitted, provided the original work is properly cited. 
This matrix element at zero momentum transfer is fixed by the fact, that the interaction is due to the local $\mathrm{U}(1)$ symmetry, whose global counterpart is producing the conserved charge (and of course is depending on the normalization of eigenvectors $\left.\left\langle P \mid P^{\prime}\right\rangle=(2 \pi)^{3} 2 E \delta\left(\vec{P}-\vec{P}^{\prime}\right)\right)$.

$$
\left\langle P\left|J_{q}^{\mu}\right| P\right\rangle=2 e_{q} P^{\mu}
$$

so that in the rest frame the interaction is completely defined by the scalar potential

$$
M_{0}=\left\langle P\left|J_{q}^{\mu}\right| P\right\rangle A_{\mu}=2 e_{q} M \phi
$$

At the same time, the interaction with the weak classical gravitational field is:

$$
M=\frac{1}{2} \sum_{q, G}\left\langle P^{\prime}\left|T_{q, G}^{\mu}\right| P\right\rangle h_{\mu \nu},
$$

where $h$ is a deviation of metric tensor from its Minkowski value. The relative factor $1 / 2$, which will play a crucial role, is coming from the fact, that the variation of the action with respect to the metric is producing an energy-momentum tensor with the coefficient $1 / 2$, while the variation with respect to classical source $A^{\mu}$, is producing the current without such a coefficient. It is this coefficient, that guarantee the correct value for the Newtonian limit, fixed by the global translational invariance

$$
\sum_{q, G}\left\langle P\left|T_{i}^{\mu \nu}\right| P\right\rangle=2 P^{\mu} P^{\nu},
$$

which, together with the approximation for $h$ (with factor of 2 having the geometrical origin $)^{2}$

$$
h_{00}=2 \phi(x)
$$

is resulting in the rest frame expression

$$
M_{0}=\sum_{q, G}\left\langle P\left|T_{i}^{\mu \nu}\right| P\right\rangle h_{\mu \nu}=2 M \cdot M \phi,
$$

where we used the same notation for gravitational and scalar electromagnetic potentials, and identified normalization factor $2 M$ in order to make the similarity between (3) and (7) obvious. One can see that the interaction with gravitational field is described by the charge, equal to the particle mass, which is just the equivalence principle. It is appearing here as low energy theorem, rather than postulate. The similarity with electromagnetic case allows one to clarify the origin of such a theorem, suggesting, that the interaction with gravity is due to the local counterpart of global symmetry, although it may be proved starting just from the Lorentz invariance of the soft graviton approximation. ${ }^{3}$

The situation with the terms linear in $\Delta$ is different for electromagnetism and gravity. While such a term is defined by the specific dynamics in the electromagnetic case, producing the anomalous magnetic moment, the similar contribution in the gravitational case is entirely fixed by the angular momentum conservation, 
which was known in the context of gravity for many years since the ingenious observation of Kobzarev and Zakharov ${ }^{4}$ and developed later. ${ }^{5,6}$, a It means, in terms of the gravitational interaction, that (straightforwardly generalizing the pioneering result $^{7}$ ) Anomalous Gravitomagnetic Moment (AGM) of any particle is identically equal to zero.

The similar effects for Dirac equation was considered by Yuri Obukhov, Alexander Silenko and myself (see e.g. Ref. 8)

Let us clarify this statement, ${ }^{1}$ which is not restricted to the nucleon or spin- $1 / 2$ Dirac particle. The presence of Dirac spinors in the parametrization of gravitational form factors is actually not crucial. To show that, it is convenient to use the equation of motion in order to attribute all the $\Delta$-dependence to the anomalous form factor $P^{\mu} \bar{u} \sigma^{\nu \alpha} u \Delta_{\alpha}$. As soon as the linear $\Delta$-dependence is already extracted, the spinors can be taken at the same momentum, which is convenient to choose as an average one $P$, and calculation of the matrix element is reduced to the trace of density matrix

$$
\begin{aligned}
\bar{u}(P) \sigma^{\nu \alpha} \Delta_{\alpha} u(p) & =\operatorname{Tr} \rho(P) i \sigma^{\nu \alpha} \Delta_{\alpha} \\
& =\operatorname{Tr} \frac{1}{2}(\hat{P}+M)\left(1+\hat{S} \gamma_{5}\right) i \sigma^{\nu \alpha} \Delta_{\alpha}=2 i \epsilon^{\rho \sigma \nu \alpha} P^{\rho} S^{\sigma} \Delta^{\alpha}
\end{aligned}
$$

The constraint for AGM of Dirac fermion by considering the matrix element of the projection of Pauli-Lubanski operator, may be now easily generalized to the particle of any spin, so that, for the total conserved energy momentum tensor of all the constituents is (c.f. Ref. 9)

$$
\left\langle P^{\prime}\left|\sum T^{\mu \nu}\right| P\right\rangle=2 P^{\mu} P^{\nu}+i P^{(\mu} \epsilon^{\nu) \sigma \rho \alpha} P^{\rho} S^{\sigma} \Delta^{\alpha} / M
$$

Like in the spin $1 / 2$ case, $S$ is the average spin (whose appearance is dictated by the positive charge parity in tyhe $t$-channel) in any of the states $|P>,| P^{\prime}>$ (the difference between which is inessential, as soon as linear terms in $\Delta$ are considered, and we postpone the discussion of the spin-flip case).

As soon as the form factors in spin- $1 / 2$ case differ from the ones for the matrix element of vector current $J^{\mu}$ by the common factor $P^{\nu}$, one may define gyrogravitomagnetic ratio in the same way as common gyromagnetic ratio, and it should have Dirac value $g=2$ for particle of any $\operatorname{spin} J$

$$
\mu_{G}=J
$$

which coincide with the standard Dirac magnetic moment, up to the interchange $e \leftrightarrow M$, making the Bohr magneton equal to $1 / 2$.

However, the situation changes if one define the gyrogravitomagnetic moment as a response to the external gravitomagnetic field. The $\epsilon$ tensor in the coordinate space produce the curl, and the gravitomagnetic field, acting on the particle spin,

aThe reason is that the structure of Poincare group is more rich than that of $U(1)$ group. 
is equal to

$$
\vec{H}_{J}=\frac{1}{2} \operatorname{rot} \vec{g} ; \quad \vec{g}_{i} \equiv g_{0 i},
$$

where factor $1 / 2$ is just the mentioned normalization factor in (6). The relevant off-diagonal components of the metric tensor may be generated by the rotation of massive gravity source. ${ }^{2}$

There is also another effect, induced by this field: the straightforward analog of Lorentz force, ${ }^{2}$ produced by the first (spin-independent) term in (10). In that case the gravitomagnetic filed, for the low velocity of the particle (such a restriction is actually inessential, as we can always perform the Lorentz boost, making the particle velocity small enough) is

$$
\vec{H}_{L}=\operatorname{rot} \vec{g}=2 \vec{H}_{G},
$$

Consider now the motion of the particle in the gravitomagnetic field. The effect of Lorentz force is reduced, due to the Larmor theorem, (which is also valid for small velocity) to the rotation with the Larmor frequency

$$
\omega_{L}=\frac{H_{L}}{2} .
$$

This is also the frequency of the macroscopic gyroscope dragging. At the same time, the microscopic particle dragging frequency is

$$
\omega_{J}=\frac{\mu_{G}}{J} H_{J}=\frac{H_{L}}{2}=\omega_{L}
$$

The common frequency for microscopic and macroscopic gyroscope is just the Larmor frequency, so that the gravitomagnetic field is equivalent to the frame rotation. This should be considered as a Post-Newtonian manifestation of the equivalence principle.

\section{Extension of Equivalence Principle}

We are going to discuss the suggestion ${ }^{10}$ that contributions of quarks and gluons to the Anomalous Gravitomagnetic Moment of nucleon are zero in NPQCD,

$$
\left\langle P^{\prime}\left|T_{i}^{\mu \nu}\right| P\right\rangle=N_{i}\left[2\left(P^{\mu} P^{\nu}-\frac{g^{\mu \nu}}{4} M^{2}\right)+\frac{i}{M} P^{(\mu} \epsilon^{\nu) \sigma \rho \alpha} P^{\rho} S^{\sigma} \Delta^{\alpha}\right]+O\left(g^{\mu \nu}, \Delta^{2}\right) .
$$

To test this suggestion let us first turn to perturbative QED analysis. The matrix elements of energy momentum tensors of electrons and photons acquire the logarithmically divergent contributions, canceled in their sum. This problem, at leading order(LO), is similar to the calculation of QED corrections to gravity coupling. ${ }^{11}$ It is sufficient to consider the matrix elements of either electron or photon energy momentum tensor switched between free electron states, and the latter case is more simple, being described by the single diagram. It is enough to consider the terms of 
zero and first order in $\Delta$. The divergent contribution to the former is appearing ${ }^{11}$ in the traceless part and may be identified with the second moment of spin-independent DGLAP kernel $\int_{0}^{1} d x x P_{G q}(x)$. The linear term is known from the orbital angular momentum calculations ${ }^{12-14}$ and is also equal to that quantity, so that AGM is really zero.

The next important step is to consider the respective finite terms, constituting the AGM of electron. It was calculated in QED, ${ }^{15,16}$ resulting in the check of the lowenergy theorems. To consider the separate AGM of photons and gluons in photon, one should perform the respective decomposition of the total result, and get the non-zero answer, confirmed later by S. Brodsky and collaborators. ${ }^{17}$

The general reason for this value was also founded ${ }^{15}$ as emerging due to use the unsubtracted dispersion relations for the relevant form factors, while performing the subtraction, resulting in the zero AGM, leads to the absence of the smooth transition $m_{e} \rightarrow 0$. $^{\mathrm{b}}$

Performing these subtraction for the separate contributions of various fields while imposing Extended Equivalence Principle (ExEP) means, that such a gauge invariance is postulated for each field separately. The QED example shows, that due to fields interactions the simplest form of such assumption is not valid. However, we will now present some arguments in favor of the hypothesis, ${ }^{18}$ that in full QCD manifesting such phenomena as confinement and chiral symmetry breaking, separate gauge invariance of quarks and gluons (and, therefore, EEP and equipartition) may be restored.

Note that due to field interactions the scattering of electron in the external gravitational field is accompanied by the emission of soft photons. Let us therefore suggest, that EEP should include this emitted photons, and the zero AGM should correspond to the "coherent state" of "bare" electron (which is the QED analog of quark contribution to the nucleon AGM) and emitted photon. In QCD, due to confinement phenomenon, the emission of soft gluons is forbidden and zero AGM of quarks themselves should appear. Note that only the singlet combination of quarks could manifest zero AGM, which is seen already from the perturbative evolution: ${ }^{14}$ only the total contribution of all quarks, appearing as virtual in QCD beta-function, can preserve equipartition at various $Q^{2}$. The equipartition therefore holds at leading order both in QED and QCD. At NLO order it is violated in QED, and, according to our hypothesis, this corresponds to the modification of EEP by including the soft emitted photons. In QCD equipartition is, in the same line of reasoning, restored due to confinement, so that same phenomenon is manifested at LO and beyond the perturbation theory.

The confinement phenomenon is accompanied by chiral symmetry restoration, which, according to present understanding, ${ }^{19}$ is deeply related to confinement and, in particular, happens at the same temperature in the lattice simulations. This makes the mentioned above argument ${ }^{15}$ against the subtraction, providing the

${ }^{\mathrm{b}} \mathrm{I}$ am indebted to K. Milton for the illuminating correspondence concerning this issue. 
equipartition, unapplicable to full QCD. Indeed, the term $1 / m_{q}$ provided by this subtraction in perturbation theory should be replaced by hadronic scale ${ }^{c}$ when chiral symmetry breaking is taken into account.

\section{Equivalence Principle for Vector Mesons}

It leads ${ }^{20}$ to zero sum rule for the sum of second moments of quark and gluon tensor spin structure functions derived many years ago, ${ }^{21,22}$ and the suggested manifestation of EP is related to them like that of Kobzarev and Okun is related to Ji's Sum Rules.

$$
\begin{gathered}
\sum_{q}\left\langle P, S\left|T_{i}^{\mu \nu}\right| P, S\right\rangle_{\mu^{2}}=2 P^{\mu} P^{\nu}\left(1-\delta\left(\mu^{2}\right)\right)+2 M^{2} S^{\mu \nu} \delta_{1}\left(\mu^{2}\right) \\
\left\langle P, S\left|T_{g}^{\mu \nu}\right| P, S\right\rangle_{\mu^{2}}=2 P^{\mu} P^{\nu} \delta\left(\mu^{2}\right)-2 M^{2} S^{\mu \nu} \delta_{1}\left(\mu^{2}\right)
\end{gathered}
$$

ExEP, in turn, means that it is valid separately for quarks and for gluons so that $\delta\left(\mu^{2}\right) \approx 0$. The available data on deuteron tensor spin structure function ${ }^{23}$ provide just the required singlet combination for quarks and indicate that second moment is compatible to zero better than the first one. This means that collective gluons in deuteron are suppressed in comparison to collective sea and ExEP provides the explanation of this fact.

The QCD Sum Rules calculations allow to test ExEP also for vector $(\rho)$ mesons. The recent observation ${ }^{24}$ of the very different behaviour of quark distributions in longitudinally and transversely polarized mesons means that tensor polarization dependent distribution crosses zero at two points.

This is the necessary condition for the function to have two zero moments and when it was found in our paper ${ }^{21}$ (in which we in fact neglected sea quarks, making extra difference between first and second moments), AV suggested a nice mechanical analogy with positions of center of mass and center of inertia, which was certainly related with his experience of teaching the classical mechanics to engineers.

At the same time, the direct calculation ${ }^{25}$ of the quark energy-momentum tensors in longitudinally and transverse polarized mesons lead to the different values, so that further investigation is of interest.

Recently, the gyromagnetic ratio of charged $\rho$ mesons was calculated in lattice $\mathrm{QCD}^{26}$ and the value fairly close to ExEP supported factor 2 was obtained.

\section{AdS/QCD and GPD/TMD Relation}

Recently $^{27}$ in the framework of AdS/QCD exloring the duality to the effective extra-dimensional gravity the (approximate) relation between GPDs and TMDs was obtained

$$
\frac{\partial}{\partial|t|}[\ln (\mathrm{GPD})]=\frac{(1-x)^{2}}{4} \frac{\partial}{\partial p_{\perp}^{2}}[\ln (\mathrm{TMD})]
$$

${ }^{\mathrm{c}}$ More specifically, by the ratio of gluon and quark condensates, determined by trace anomaly. 
The universality in the $t$ and $p_{\perp}^{2}$ dependence of the GPDs and TMDs comes from the AdS/QCD wave function. The GPDs obtained from the model satisfy the Regge behavior with the Regge slope $\alpha^{\prime}=1 /\left(4 \kappa^{2}\right)$. The average value of Gaussian transverse momentum distribution is then obtained as

$$
\left\langle p_{\perp}^{2}\right\rangle=\frac{(1-x)^{2}}{4 \alpha^{\prime} \ln 1 / x}=\frac{\kappa^{2}(1-x)^{2}}{\ln 1 / x},
$$

which takes the maximum value of $\left\langle p_{\perp}^{2}\right\rangle_{\max } \sim(250 \mathrm{MeV})^{2}$ for $x \sim 1 / 3$.

\section{Cosmological Implications of Extended Equivalence Principle}

One may of course think that separate smallness of quark and gluon AGM for nucleons and closeness of quark gravigyromagnetic ratio to 2 for vector mesons is occasional. It is possible nevertheless to speculate about their possible relevance to very general problems of gravity and cosmology.

Consider very strong gravity field near the Black Hole. Assume also the semiclassical picture with average angular momenta of quarks and gluons precessing independently. If their angular velocities are different the field may deconfine hadrons. If, however, they precess in accordance due to ExEP, the confinement may be gravityproof, which is attracting due to famous Black Hole complementarity. ${ }^{28}$

Another possibility may be the closeness of ExEP values to asymptotic ones. Assuming that QCD evolution is gowing backward in the expanding Universe one should recall that this process is "antikinetic". ${ }^{29}$ As a result, too large deviations from asymptotic values at large scales should result in the violation of positivity at lower scales. Assuming the existing of Multiverse one may expect that probability to get the particular deviation from the asymptotic values should be its decreasing function. As a result, Vilenkin's Mediocrity Principle (see e.g. Ref. 30) should favor the smaller deviations from asymptotic values.

\section{Conclusions}

The interaction of hadrons with external electromagnetic and gravity fields opens an interesting possibility of validity of Extended Equivalence Principle.

\section{Acknowledgments}

I am grateful to Organizers to warm hospitality. This work supported in part by RFBR Grant 14-01-00647.

\section{References}

1. O. V. Teryaev, hep-ph/9904376.

2. L. D. Landau and E. M. Lifshitz, Theoretical Physics, v.2.

3. S. Weinberg, Phys. Rev. 135 (1964) B1049.

4. I. Yu. Kobzarev and V. I. Zakharov, Annals of Phys. 37, 1 (1966). 
5. D. G. Boulware, S. Deser, Ann. Phys. 89 (1975).

6. C. F. Cho and N. D. Hari Dass, Phys. Rev. D 14 (1976) 2511.

7. I. Y. Kobzarev and L. B. Okun, Zh. Eksp. Teor. Fiz. 43 (1962) 1904 [Sov. Phys. JETP 16 (1963) 1343].

8. Y. N. Obukhov, A. J. Silenko and O. V. Teryaev, Phys. Rev. D 80 (2009) 064044 [arXiv:0907.4367 [gr-qc]].

9. R. L. Jaffe and A. Manohar, Nucl. Phys. B 337 (1990) 509; Phys. Rev. Lett. 61 (1988) 2639; Phys. Lett. A 173 (1993) 347; Nuovo Cim. B 111 (1996) 855 and Ref. therein; Murthy, Class. Quant. Grav. 6 (1989) L167.

10. O. V. Teryaev, Czech. J. Phys. 53 (2003) 47 [hep-ph/0306301].

11. J. F. Donogue, B. Holstein and R. Robinett, Phys. Rev. D 30 (1984) 2561.

12. X. Ji, J. Tang and P. Hoodbhoy, Phys. Rev. Lett. 76 (1996) 740; X. Ji, Phys. Rev. Lett. 78 (1997) 610; UMD-PP-97-042, Oct 1996. Presented at 12th International Symposium on High-energy Spin Physics (SPIN96), Amsterdam,

13. P. Hägler and A. Schäfer, hep-ph/9802362. Phys. Lett. B 430 (1998) 179.

14. O. V. Teryaev, hep-ph/9803403.

15. K. Milton, Phys. Rev. D 4 (1971) 3579; D15 (1977) 538.

16. F. A. Berends and R. Gastmans, Ann. Phys. 98 (1976) 225.

17. S. J. Brodsky, D. S. Hwang, B. Q. Ma and I. Schmidt, Nucl. Phys. B 593, 311 (2001).

18. O. V. Teryaev, "Spin structure of nucleon in QCD: Inclusive and exclusive processes," http://www.slac.stanford.edu/spires/find/hep/www?irn=4868722SPIRES entry, Prepared for International Conference on New Trends in High-Energy Physics: Experiment, Phenomenology, Theory, Yalta, Crimea, 22-29 Sep 2001

19. P. de Forcrand and M. D'Elia, Phys. Rev. Lett. 82, 4582 (1999).

20. O. V. Teryaev, Mod. Phys. Lett. A 24 (2009) 2831.

21. A. V. Efremov and O. V. Teryaev, Sov. J. Nucl. Phys. 36, 557 (1982) [Yad. Fiz. 36, $950(1982)]$.

22. A. V. Efremov and O. V. Teryaev, arXiv:hep-ph/9910555.

23. A. Airapetian et al. [HERMES Collaboration], Phys. Rev. Lett. 95, 242001 (2005) [arXiv:hep-ex/0506018].

24. A. G. Oganesian, Phys. Atom. Nucl. 71, 1439 (2008) [arXiv:hep-ph/0610090].

25. A. Oganesian and A. Samsonov, JHEP 0109, 002 (2001) [arXiv:hep-ph/0107077].

26. E. V. Luschevskaya, O. A. Kochetkov, O. V. Teryaev and O. E. Solovjeva, JETP Lett. 101 (2015) 10, 674.

27. T. Maji, C. Mondal, D. Chakrabarti and O. V. Teryaev, arXiv:1506.04560 [hep-ph].

28. L. Susskind, L. Thorlacius and J. Uglum, Phys. Rev. D 48 (1993) 3743 [hepth/9306069].

29. X. Artru, M. Elchikh, J. M. Richard, J. Soffer and O. V. Teryaev, Phys. Rept. 470 (2009) 1 [arXiv:0802.0164 [hep-ph]].

30. A. Vilenkin, arXiv:1108.4990 [hep-th]. 